\title{
High cytomegalovirus serology and subsequent COPD-related mortality: a longitudinal study
}

\author{
Raffaella Nenna $\mathbb{1}^{1,2}$, Jing Zhai ${ }^{1}$, Samuel E. Packard ${ }^{1}$, Amber Spangenberg ${ }^{1}$, \\ Duane L. Sherrill ${ }^{1}$, Fernando D. Martinez ${ }^{1}$, Marilyn Halonen ${ }^{1,3}$ and \\ Stefano Guerra ${ }^{1,4}$
}

Affiliations: ${ }^{1}$ Asthma and Airway Disease Research Center, University of Arizona, Tucson, AZ, USA. ${ }^{2}$ Dept of Pediatrics, "Sapienza" University of Rome, Rome, Italy. ${ }^{3}$ Dept of Pharmacology, College of Medicine, University of Arizona, Tucson, AZ, USA. ${ }^{4}$ ISGlobal, Barcelona Institute for Global Health, Barcelona, Spain.

Correspondence: Stefano Guerra, Asthma and Airway Disease Research Center, University of Arizona, 1230 N. Cherry Ave, Biosciences Research Laboratories Building, Tucson, AZ 85721, USA. E-mail: stefanodemail. arizona.edu

\section{ABSTRACT}

Background: Positive serology for cytomegalovirus (CMV) has been associated with all-cause mortality risk but its role in COPD mortality is unknown. The objective of the present study was to assess the relationship between CMV serology and COPD mortality.

Methods: We analysed data from 806 participants in the Tucson Epidemiological Study of Airway Obstructive Disease who, at enrolment, were aged 28-70 years and had completed lung function tests. We tested CMV serology in sera from enrolment and defined "high CMV serology" as being in the highest tertile. Vital status, date and cause of death were assessed through death certificates and/or linkage with the National Death Index up to January 2017. The association of CMV serology with all-cause and causespecific mortality risk was tested in Cox models adjusted for age, sex, level of education, body mass index, smoking status and pack-years.

Results: High CMV serology was marginally associated with all-cause mortality $(p=0.071)$ but the effect was inversely dependent on age, with the association being much stronger among participants $<55$ years than among participants $\geqslant 55$ years at enrolment ( $p$-value for CMV-by-age interaction $<0.001$ ). Compared with low CMV serology, high CMV serology was associated with mortality from COPD among all subjects (adjusted hazard ratio (HR) 2.38, 95\% CI 1.11-5.08; $\mathrm{p}=0.025$ ) and particularly in subjects $<55$ years old at enrolment (HR 5.40, 95\% CI 1.73-16.9; p=0.004). Consistent with these results, high CMV serology also predicted mortality risk among subjects who already had airflow limitation at enrolment (HR 2.10, 95\% CI 1.20-3.68; $\mathrm{p}=0.009$ ).

Conclusions: We report a strong relationship between CMV serology and the risk of dying from COPD, and thus identify a novel risk factor for COPD mortality.

@ERSpublications

Using a 45-year longitudinal population-based cohort, it was demonstrated for the first time that high CMV serology predicts COPD mortality risk, particularly in younger subjects, identifying a novel and early risk factor for COPD mortality http://bit.ly/32odP0Q

Cite this article as: Nenna R, Zhai J, Packard SE, et al. High cytomegalovirus serology and subsequent COPD-related mortality: a longitudinal study. ERJ Open Res 2020; 6: 00062-2020 [https:// doi.org/10.1183/23120541.00062-2020].

This article has supplementary material available from openres.ersjournals.com

Received: 10 Feb 2020 | Accepted: 18 Feb 2020

Copyright $\odot$ ERS 2020. This article is open access and distributed under the terms of the Creative Commons Attribution Non-Commercial Licence 4.0. 


\section{Introduction}

Cytomegalovirus (CMV) is a highly transmissible $\beta$-herpesvirus with a broad cellular tropism. The global CMV seroprevalence is estimated to be $83 \%$, although estimates vary remarkably across geographic regions and age distributions [1]. Data from the cross-sectional National Health and Nutrition Examination Survey study in the USA showed that older age, female sex, ethnicity and low socioeconomic status were independently associated with CMV seropositivity [2].

After the first CMV infection, which is generally asymptomatic, the virus is never cleared from the host. This pathogen may persist in many tissues, from which it is shed intermittently, and it establishes a lifelong latent/persistent infection with periodic subclinical reactivations. Once seropositivity is established, antibody titres raise during reactivations [3]. While in the past, CMV was thought to peacefully cohabit within immunocompetent subjects, more recently, it has been demonstrated that frequent reactivations are associated with higher levels of proinflammatory cytokines and, consequently, chronic inflammation $[4,5]$. Moreover, CMV infection and the related alterations in circulating immune cell subsets [6] are consistent with reduction in the ability of the host to fight new infections [7].

Multiple [8-15] - although not all [16, 17] - previous studies have reported that individuals with positive CMV serology are at increased risk for all-cause mortality. Studies that have looked at the relationship of CMV with cause-specific mortality have mainly focused on cardiovascular outcomes, with inconsistent results $[8,9,11-13]$.

Although a recent study has shown that individuals with chronic obstructive pulmonary disease (COPD) have increased levels of IgG against CMV [18], to date, CMV serology has not been reported as being associated with COPD mortality. COPD is characterised by airway damage and irreversible airflow limitation [19] that is related to either an accelerated decline of lung function in adulthood, or to tracking of lung function deficits from young to late adult life [20]. Both systemic inflammation [21] and recurrent infections [22] are thought to play pivotal roles in COPD.

In this study, using a large population-based prospective cohort with up to 45 years of follow-up, we sought to examine the association of baseline CMV serology to subsequent COPD-related mortality.

\section{Methods}

\section{Participants}

We used data from the Tucson Epidemiological Study of Airway Obstructive Disease (TESAOD). TESAOD is a population-based prospective cohort study of non-Hispanic white households in Tucson, AZ, USA [23] that was initiated in 1972. At enrolment, participants completed a standardised respiratory questionnaire and lung function tests, and research nurses measured height and weight, and obtained blood samples.

\section{Lung function at enrolment}

Lung function measurements included pre-bronchodilator forced expiratory volume in $1 \mathrm{~s}\left(\mathrm{FEV}_{1}\right)$, forced vital capacity $(\mathrm{FVC})$, the $\mathrm{FEV}_{1} / \mathrm{FVC}$ ratio and forced expiratory flow at $25-75 \%$ of $\mathrm{FVC}\left(\mathrm{FEF}_{25-75 \%}\right)$. Per cent predicted values for $\mathrm{FEV}_{1}, \mathrm{FVC}$ and $\mathrm{FEF}_{25-75 \%}$ were computed using reference equations generated in the same population by KNUDSON et al. [24].

Airflow limitation was defined as $\mathrm{FEV}_{1} / \mathrm{FVC}$ ratio $<70 \%$.

\section{Vital status}

Vital status was updated during the study follow-up through direct contact with family and next of kin, and collection of death certificates. In addition, a systematic review of mortality as of 1 January 2017 was completed through linkage with the National Death Index. Dates and causes of death were determined based on death certificates for events up to 1978 and from National Death Index records for events after 1978. For this study, we analysed three causes of death: COPD (International Classification of Diseases (ICD)-9: 490-494 and 496; ICD-10: J40-J47), cardiovascular disease (ICD-9: 390-459; ICD-10: I00-I99) and cancer (ICD-9: 140-208 and 238.6; ICD-10: C00-C97).

\section{Molecular assays}

Cryopreserved serum samples from enrolment were analysed at the Myriad-RBM laboratory (Austin, TX) using the Human MAP infectious panel, a bead-based suspension multiplex assay based on Luminex immunoassay technology. For this study, we used data on serology against CMV and against two bacteria with tropism for the lung, which were included in the same infectious panel and have been previously implicated in COPD: Chlamydophila pneumoniae and Mycoplasma pneumoniae. Serology results were calculated as a ratio of the median fluorescence intensity (MFI) of target-specific, antigen-coupled 
microspheres to MFI generated by a negative control microsphere tested in each sample well. The CMV serology was validated with a single CMV ELISA (SERION ELISA classic CMV IgG; QED Bioscience Inc., San Diego, CA, USA) on a subgroup of 64 serum samples randomly selected from the 806 TESAOD participants. We found correlation of measurement results of the two methods (Spearman's $\rho=0.64$, $\mathrm{p}<0.0001)$.

C-reactive protein (CRP) levels were measured in the same 806 serum samples using the enzymatic solid-phase chemiluminescent immunometric assay performed on the Immulite 2000 (Siemens Diagnostics, Tarrytown, NY, USA).

\section{Statistical analysis}

The distributions of CMV, C. pneumoniae and M. pneumoniae serology, and CRP values were skewed (long tail to the right) and, therefore, we used logarithmic transformation to obtain normalisation. Values were then standardised to z-scores to allow testing of effects associated with 1-sD increase. For main analyses, CMV, C. pneumoniae and M. pneumoniae serology values were categorised into tertiles (low, medium and high) to investigate the association of high serology with mortality risk.

Survival curves and Cox proportional-hazards models were used to study the effects of CMV serology and other covariates on all-cause and cause-specific mortality, after adjustment for age, sex, level of education, body mass index, smoking status and pack-years. The main results were confirmed in sensitivity analyses that included a batch indicator for CMV assays as additional covariate. Time to event was defined as the years from enrolment to either the date of death for deceased participants or to 1 January 2017 for subjects who were still alive at that time.

For cause-specific mortality analyses, we considered COPD and the other two leading causes of death: cardiovascular disease and cancer. In Cox models for each of these diseases, cases were represented by participants who had that specific disease as the underlying cause of death listed on death certificate (participants who "died from" that disease). In secondary Cox models, we evaluated cause-specific mortality by defining cases as events with the specific disease indicated as either underlying cause of death or co-existing condition on the death certificate (participants who "died from or with" that disease). Death events due to causes other than the specific cause of interest were treated as censored observations, but the main results were confirmed using competing risk models. In age-stratified analyses, subjects were divided into those $<55$ and those $\geqslant 55$ years of age at enrolment. The cut-off at 55 years was based on the interaction between CMV and age observed in the main Cox model on all-cause mortality (see Results section). Stata 15.1 (StataCorp, College Station, TX, USA) and R 3.4.1 (The R Foundation for Statistical Computing, Vienna, Austria) were used for analysis.

\section{Results}

For this study, we used data from 806 participants who, at enrolment, were aged 28-70 years, had serum samples available and had completed lung function tests. These 806 participants were older, more frequently women and asthmatics, and less frequently current smokers as compared with the 28-70-year-old TESAOD participants $(n=986)$ who were excluded from this study (mainly because of serum availability) (table $\mathrm{S} 1$ ).

At enrolment, the mean age \pm SD of included participants was $53 \pm 12$ years; 485 (60.2\%) were females and $116(14.4 \%)$ had airflow limitation. A total of 503 participants (62.5\%) were ever- smokers (222 former and 281 current smokers) with $29 \pm 22$ pack-years. CMV serology was positively associated with age, female sex, a lower level of education, obesity and indices of airflow limitation (table 1). Significant correlations were observed for CMV serology with pack-years, C. pneumoniae and M. pneumoniae serology, and CRP levels. After adjusting for age, sex and level of education, all these associations were confirmed, except for the association between CMV and pack-years, which was mostly confounded by age (data not shown).

\section{High CMV serology and all-cause mortality}

During the 45-year follow-up, 668 (82.9\%) out of 806 participants died. A total of 268 (66.5\%) out of 403 participants aged $<55$ years at enrolment died during follow-up, as did 400 (99.3\%) out of 403 participants aged $\geqslant 55$ years.

After adjusting for sex, age, body mass index (BMI), level of education, smoking status and pack-years, subjects in the highest tertile for CMV serology (hereafter referred to as high CMV serology) had a $21 \%$ increase in all-cause mortality risk compared to subjects in the lowest tertile, although the association was of borderline significance (table 2 and figure 1a). However, there was a highly significant CMV-by-age interaction, indicating that the relation of CMV serology to mortality decreased as age at enrolment increased ( $p$-value for interaction $<0.001$ ), with significant effects of CMV on all-cause mortality up to 


\begin{tabular}{|c|c|c|c|}
\hline & Participants & $\beta$-coefficient $(95 \% \mathrm{CI})$ & p-value \\
\hline Age in years & 806 & $0.025(0.02-0.03)$ & $<0.001$ \\
\hline Sex (ref.: male) & & & $<0.001$ \\
\hline Female & $485 / 806$ & $0.27(0.13-0.41)$ & \\
\hline Education level (ref.: >12 years) & & & $<0.001$ \\
\hline$\leqslant 12$ years & $496 / 806$ & $0.33(0.19-0.47)$ & \\
\hline \multicolumn{4}{|l|}{ BMI category (ref.: normal weight) } \\
\hline Underweight & 15/784 & $0.21(-0.31-0.72)$ & 0.427 \\
\hline Overweight & $285 / 784$ & $0.02(-0.13-0.17)$ & 0.768 \\
\hline Obese & $65 / 784$ & $0.33(0.06-0.59)$ & 0.014 \\
\hline \multicolumn{4}{|l|}{ Smoking status (ref.: never) } \\
\hline Former & $222 / 805$ & $-0.12(-0.29-0.06)$ & 0.189 \\
\hline Current & $281 / 805$ & $-0.01(-0.17-0.16)$ & 0.943 \\
\hline \multicolumn{4}{|l|}{ Airflow limitation" (ref.: stage 0) } \\
\hline Stage 1 & $43 / 806$ & $0.13(-0.18-0.44)$ & 0.400 \\
\hline Stage 2 & $56 / 806$ & $0.21(-0.06-0.48)$ & 0.130 \\
\hline Stage $3+4$ & $17 / 806$ & $0.57(0.08-1.05)$ & 0.021 \\
\hline \multicolumn{4}{|l|}{ Doctor-diagnosed asthma (ref.: no) } \\
\hline Yes & $97 / 806$ & $0.13(-0.09-0.34)$ & 0.242 \\
\hline $\mathrm{FEV}_{1} \%$ predicted & 806 & $-0.005(-0.008--0.001)$ & 0.011 \\
\hline FVC $\%$ predicted & 806 & $-0.003(-0.007-0.001)$ & 0.116 \\
\hline $\mathrm{FEV}_{1} / \mathrm{FVC}$ ratio & 806 & $-0.012(-0.019--0.005)$ & 0.001 \\
\hline FEF $_{25-75 \%} \%$ predicted & 806 & $-0.002(-0.004-0.0003)$ & 0.022 \\
\hline \multicolumn{4}{|l|}{ Smoking history pack-years } \\
\hline All & 805 & $0.003(0.0002-0.006)$ & 0.032 \\
\hline Only smokers & 503 & $0.007(0.003-0.10)$ & 0.001 \\
\hline Chlamydophila pneumoniae serology in z-scores & 806 & $0.17(0.10-0.24)$ & $<0.001$ \\
\hline Mycoplasma pneumoniae serology in z-scores & 806 & $0.31(0.24-0.38)$ & $<0.001$ \\
\hline C-reactive protein in z-scores & 806 & $0.14(0.07-0.21)$ & $<0.001$ \\
\hline \multicolumn{4}{|c|}{ 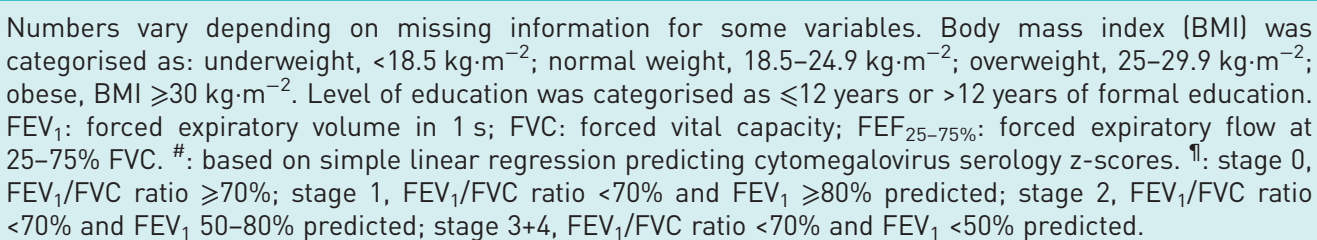 } \\
\hline
\end{tabular}

$\sim 55$ years of age at enrolment (figure 2). Consistently, when analyses were stratified according to this age cut-off, among younger subjects (<55 years), high CMV serology was associated with a striking $90 \%$ increased risk for all-cause mortality compared to low CMV serology (table 2 and figure $1 \mathrm{~b}$ ), whereas no significant effects of CMV serology were found among older subjects (table 2). Among individuals aged

TABLE 2 Associations between cytomegalovirus (CMV) tertiles and all-cause mortality in the total Tucson Epidemiological Study of Airway Obstructive Disease population and among participants who were $<55$ and $\geqslant 55$ years of age at enrolment

\begin{tabular}{|c|c|c|c|c|c|c|}
\hline & \multicolumn{2}{|c|}{$\begin{array}{c}\text { All subjects }(\mathrm{N}=783) \text {, } \\
651 \text { deaths }\end{array}$} & \multicolumn{2}{|c|}{$\begin{array}{c}<55 \text { years ( } N=387) \text {, } \\
258 \text { deaths }\end{array}$} & \multicolumn{2}{|c|}{$\begin{array}{c}\geqslant 55 \text { years }(\mathrm{N}=396), \\
393 \text { deaths }\end{array}$} \\
\hline & HR $(95 \% \mathrm{CI})$ & p-value & HR (95\% CI) & $p$-value & HR (95\% CI) & p-value \\
\hline Low CMV tertile & Ref. & & Ref. & & Ref. & \\
\hline Medium CMV tertile & $1.15(0.94-1.40)$ & 0.187 & 1.35 (0.99-1.85) & 0.059 & $0.87(0.66-1.14)$ & 0.299 \\
\hline High CMV tertile & $1.21(0.98-1.49)$ & 0.071 & $1.90(1.38-2.61)$ & $<0.001$ & $0.86(0.66-1.12)$ & 0.259 \\
\hline
\end{tabular}

Cox model adjusted for sex, age, body mass index, level of education, smoking status and pack-years. Total numbers are lower than the 806 included participants because some individuals had missing information on covariates. HR: hazard ratio. 

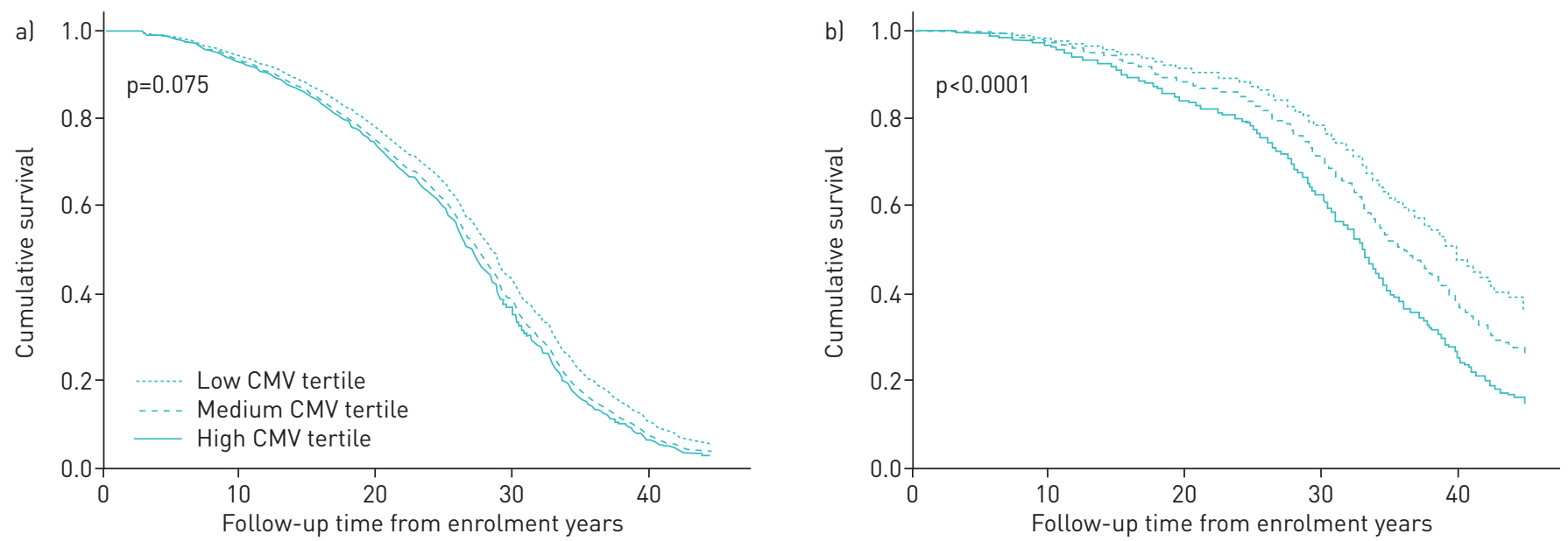

FIGURE 1 Covariate-adjusted survival curves for all-cause mortality plotted by cytomegalovirus (CMV) tertiles in a) all the 783 Tucson Epidemiological Study of Airway Obstructive Disease participants and in b) the 387 participants aged $<55$ years at enrolment. p-values refer to the trend across CMV tertiles. Total number is lower than the 806 included participants because some individuals had missing information on covariates. Curves adjusted for sex, age, body mass index (BMI), level of education, smoking status and pack-years. BMI was categorised as: underweight, $<18.5 \mathrm{~kg} \cdot \mathrm{m}^{-2}$; normal weight, $18.5-24.9 \mathrm{~kg} \cdot \mathrm{m}^{-2}$; overweight, $25-29.9 \mathrm{~kg} \cdot \mathrm{m}^{-2}$; obese, BMI $\geqslant 30 \mathrm{~kg} \cdot \mathrm{m}^{-2}$. Level of education was categorised as $\leqslant 12$ years or $>12$ years of formal education.

$<55$ years at enrolment who died during follow-up, after full adjustment, subjects with high CMV serology died, on average, 4 years earlier than those with low CMV serology $(-4.3$ years, $95 \%$ CI $-7.2--1.3$ years; $\mathrm{p}=0.004)$.

High CMV serology predicts COPD mortality

Over the follow-up period, 56 (6.9\%) out of 806 participants died from COPD and 85 (10.5\%) out of 806 died from or with COPD. After adjustment for covariates, high CMV serology was significantly associated with mortality from COPD (table 3 and figure 3a). This association was confirmed when we further adjusted the models for initial $\mathrm{FEV}_{1} \%$ predicted, indicating that CMV serology affected COPD mortality through mechanisms partly independent of initial lung function deficits. When an interaction with age was tested in Cox models, we found a trend for decreasing effects of CMV on COPD mortality with increasing age (red line in figure S1), although the CMV-by-age interaction term was not significant. Consistently, upon stratification by age, the effect of CMV on COPD mortality was strong and highly significant only in the group of participants aged $<55$ years at enrolment (table 3 and figure $3 \mathrm{~b}$ ). When we re-evaluated COPD mortality defined as either underlying cause of death or co-existing condition on the death certificate (table 3), we confirmed significant associations between CMV serology with COPD mortality, particularly among participants aged $<55$ years.

FIGURE 2 Estimated hazard ratios for the association of high cytomegalovirus (CMV) serology with all-cause mortality by age at enrolment. Estimates from Cox models adjusted for sex, age, body mass index, level of education, smoking status and pack-years, and including interaction terms between CMV serology tertiles and age at enrolment. Shading represents the $95 \%$ confidence interval.

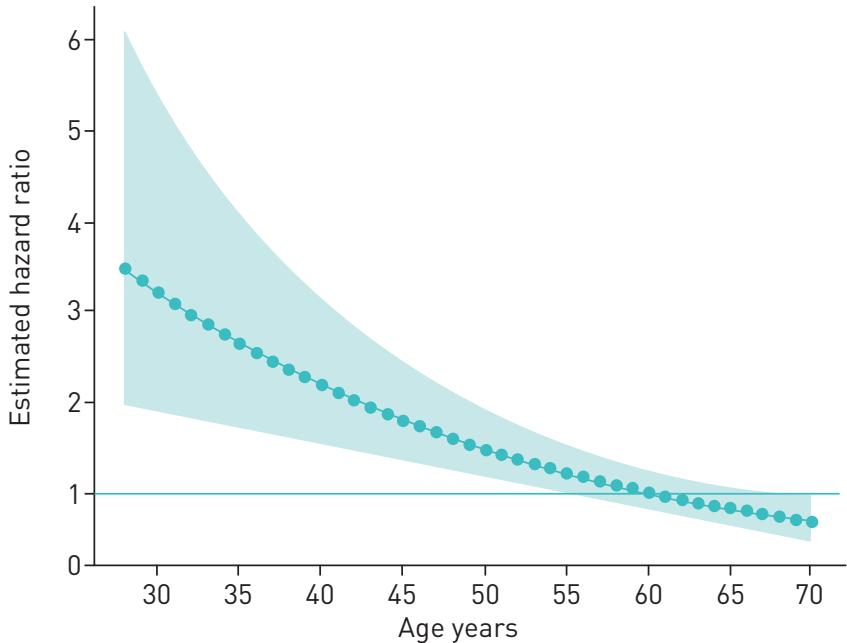




\begin{tabular}{|c|c|c|c|c|c|c|}
\hline & \multicolumn{2}{|c|}{ All subjects ( $N=783$ ) } & \multicolumn{2}{|c|}{$<55$ years $(\mathrm{N}=387)$} & \multicolumn{2}{|c|}{$\geqslant 55$ years $(N=396)$} \\
\hline & HR $(95 \% \mathrm{CI})$ & p-value & HR $(95 \% \mathrm{CI})$ & p-value & $\operatorname{HR}(95 \% \mathrm{CI})$ & p-value \\
\hline Mortality from COPD & \multicolumn{2}{|l|}{53 deaths } & \multicolumn{2}{|l|}{21 deaths } & \multicolumn{2}{|l|}{32 deaths } \\
\hline Low CMV tertile & \multicolumn{2}{|l|}{ Ref. } & \multicolumn{2}{|l|}{ Ref. } & \multicolumn{2}{|l|}{ Ref. } \\
\hline Medium CMV tertile & $1.62(0.73-3.58)$ & 0.234 & $1.28(0.33-5.02)$ & 0.721 & $1.62(0.56-4.67)$ & 0.375 \\
\hline High CMV tertile & $2.38(1.11-5.08)$ & 0.025 & $5.40(1.73-16.9)$ & 0.004 & $1.43(0.50-4.09)$ & 0.500 \\
\hline Mortality from or with COPD & \multicolumn{2}{|c|}{80 deaths } & \multicolumn{2}{|c|}{31 deaths } & \multicolumn{2}{|c|}{19 deaths } \\
\hline Low CMV tertile & \multicolumn{2}{|l|}{ Ref. } & \multicolumn{2}{|l|}{ Ref. } & \multicolumn{2}{|l|}{ Ref. } \\
\hline Medium CMV tertile & $1.58(0.82-3.06)$ & 0.171 & $1.06(0.37-3.04)$ & 0.907 & $1.95(0.75-5.07)$ & 0.169 \\
\hline High CMV tertile & $2.56(1.37-4.78)$ & 0.003 & 3.73 (1.49-9.37) & 0.005 & $2.26(0.91-5.61)$ & 0.079 \\
\hline \multicolumn{7}{|c|}{$\begin{array}{l}\text { Cox model adjusted for sex, age, body mass index, level of education, smoking status and pack-years. } \\
\text { Total number is lower than the } 806 \text { included participants because some individuals had missing } \\
\text { information on covariates. }\end{array}$} \\
\hline
\end{tabular}

Consistent with these observations, we found evidence for a dose-response relationship of CMV serology with mortality risk among participants who, at enrolment, had airflow limitation $\left(\mathrm{FEV}_{1} / \mathrm{FVC}<70 \%\right)$, the hallmark of COPD (figure S2). In this group of participants, after adjustment for covariates, subjects with medium CMV serology had a 76\% increase (hazard ratio (HR) 1.76, 95\% CI 1.02-3.06; $\mathrm{p}=0.043$ ) and subjects with high CMV serology a $110 \%$ increase (HR 2.10, 95\% CI 1.20-3.68; p=0.009) in all-cause mortality risk compared to subjects with low CMV serology.

Next, to evaluate possible effects of smoking, among the 806 participants, we stratified analyses by smoking status and found high CMV serology to be associated with increased risk of dying from or with COPD both in smokers $(n=490)$ and nonsmokers $(n=293)$ (66 deaths (HR 2.23, 95\% CI 1.16-4.31; $\mathrm{p}=0.017$ ) and 14 deaths (HR 8.89, 95\% CI 0.95-83.73; $\mathrm{p}=0.056$ ) respectively). However, the small number of COPD-related deaths among nonsmokers precluded firm conclusions on possible effect modification by smoking.

Lastly, we sought to evaluate the potential impact of $C$. pneumoniae and $M$. pneumoniae serology, and that of systemic inflammation on the association between high CMV serology and mortality from or with COPD. We did not find any of these factors to modify the effect of high CMV serology on COPD mortality
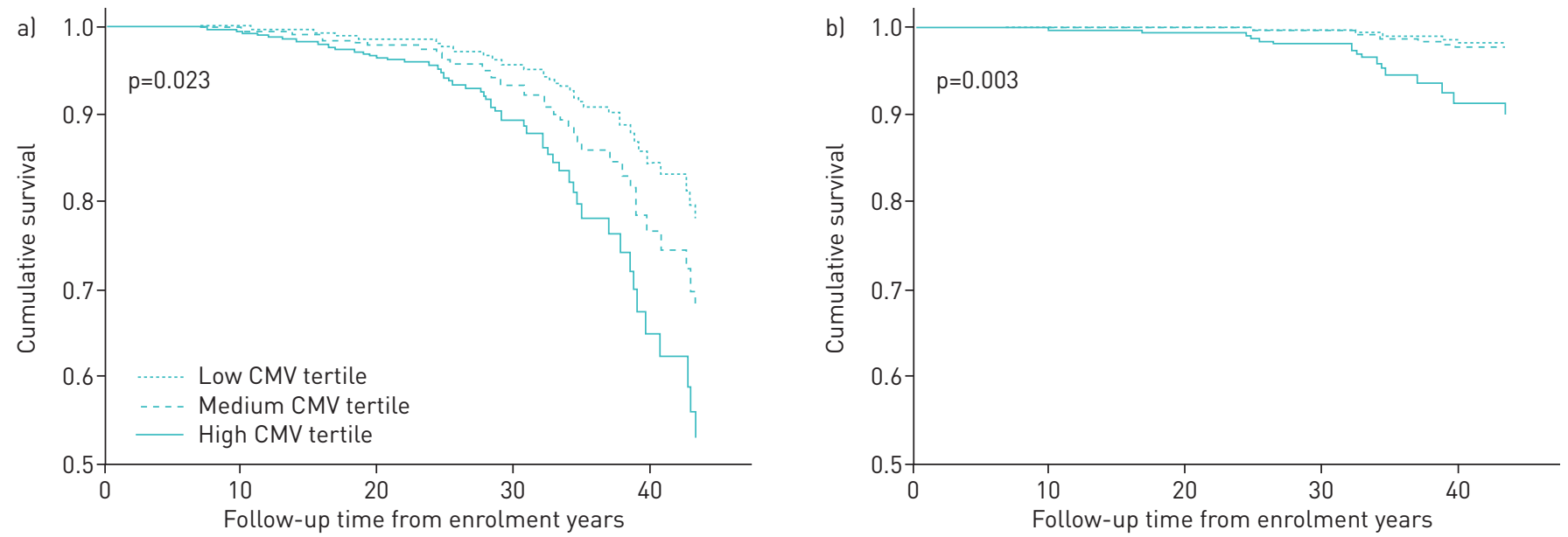

FIGURE 3 Covariate-adjusted survival curves for cause specific mortality from COPD plotted by cytomegalovirus (CMV) tertiles in a) all the 783 Tucson Epidemiological Study of Airway Obstructive Disease participants and in b) the 387 participants aged <55 years at enrolment. p-values refer to trend across CMV tertiles. Total number is lower than the 806 included participants because some individuals had missing information on covariates. Curves adjusted for sex, age, BMI, level of education, smoking status and pack-years. BMI was categorised as: underweight, $<18.5 \mathrm{~kg} \cdot \mathrm{m}^{-2}$; normal weight, $18.5-24.9 \mathrm{~kg} \cdot \mathrm{m}^{-2}$; overweight, $25-29.9 \mathrm{~kg} \cdot \mathrm{m}^{-2}$; obese, BMI $\geqslant 30 \mathrm{~kg} \cdot \mathrm{m}^{-2}$. Level of education was categorised as $\leqslant 12$ years or $>12$ years of formal education. 
(p-values for interaction of high CMV with high C. pneumoniae 0.220, with high M. pneumoniae 0.281 and with CRP 0.842). High C. pneumoniae serology ( $\mathrm{p}=0.001$ ), but not $M$. pneumoniae serology or CRP, was independently associated with increased risk for COPD mortality (table S2). In a fully adjusted Cox model (table 4) that included CMV, C. pneumoniae, M. pneumoniae, CRP, age, sex, education, BMI, smoking, pack-years and baseline $\mathrm{FEV}_{1}$, the risk for COPD mortality associated with high CMV serology, although slightly decreased, remained significant, indicating that the effects by which CMV serology increases the risk of dying of COPD are at least partly independent of immune responses to C. pneumoniae and M. pneumoniae, and of systemic inflammation.

\section{High CMV serology, and cardiovascular and cancer mortality}

Over the follow-up period, 279 (34.6\%) out of 806 participants died from cardiovascular disease and 144 (17.9\%) out of 806 from cancer. As shown in table S3, after adjusting for covariates, high CMV serology did not increase cardiovascular mortality risk. In contrast, CMV serology was associated with cancer mortality (HR 1.87, 95\% CI 1.20-2.90; p=0.006) (table S4). We found the effects of CMV on cancer mortality to strongly decrease with age (figure S1) and the corresponding CMV-by-age interaction term to be significant in Cox models ( $\mathrm{p}=0.015)$. Accordingly, upon stratification by age, the effect of high CMV on cancer mortality was significant only in the group of participants aged $<55$ years at enrolment (table S4). Among the different types of cancer, the effects of high CMV serology were particularly strong for mortality by urogenital cancer (table S5).

\section{Discussion}

Using the 45-year longitudinal TESAOD cohort, we found that high CMV serology predicts COPD mortality risk in the general adult population, even after adjustment for potential confounders. A fully adjusted model for COPD mortality revealed an association of CMV serology with the risk of dying of COPD that was independent of pre-existing lung function deficits, positive serology to the respiratory

\begin{tabular}{|c|c|c|}
\hline & $\operatorname{HR}(95 \% \mathrm{CI})$ & p-value \\
\hline \multicolumn{3}{|l|}{ CMV tertiles } \\
\hline Medium & $1.65(0.84-3.25)$ & 0.146 \\
\hline High & $2.30(1.18-4.50)$ & 0.015 \\
\hline \multicolumn{3}{|l|}{ Chlamydophila pneumoniae tertiles } \\
\hline Medium & $0.73(0.37-1.42)$ & 0.358 \\
\hline High & $2.30(1.28-4.10)$ & 0.005 \\
\hline \multicolumn{3}{|l|}{ Mycoplasma pneumoniae tertiles } \\
\hline Medium & $0.94(0.52-1.69)$ & 0.832 \\
\hline High & $0.71(0.39-1.30)$ & 0.266 \\
\hline C-reactive protein in z-scores & $1.02(0.79-1.32)$ & 0.881 \\
\hline Age in years & $1.11(1.07-1.15)$ & $<0.001$ \\
\hline Sex (ref.: male) & & 0.064 \\
\hline Female & $0.57(0.32-1.03)$ & \\
\hline Education level (ref.: $\geqslant 12$ years) & & 0.271 \\
\hline$<12$ years & $1.36(0.78-2.37)$ & \\
\hline \multicolumn{3}{|l|}{ BMI category (ref.: normal weight) } \\
\hline Underweight & $6.16(2.11-18.04)$ & 0.001 \\
\hline Overweight & $0.48(0.27-0.86)$ & 0.014 \\
\hline Obese & $0.36(0.12-1.10)$ & 0.073 \\
\hline \multicolumn{3}{|l|}{ Smoking status (ref.: never) } \\
\hline Former & $0.86(0.37-1.99)$ & 0.730 \\
\hline Current & $2.98(1.36-6.51)$ & 0.006 \\
\hline Smoking history pack-years & $1.01(1.0-1.02)$ & 0.073 \\
\hline $\mathrm{FEV}_{1} \%$ predicted & $0.95(0.93-0.96)$ & $<0.001$ \\
\hline
\end{tabular}

Body mass index (BMI) was categorised as: underweight, $<18.5 \mathrm{~kg} \cdot \mathrm{m}^{-2}$; normal weight, $18.5-24.9 \mathrm{~kg} \cdot \mathrm{m}^{-2}$; overweight, $25-29.9 \mathrm{~kg} \cdot \mathrm{m}^{-2}$; obese, $\mathrm{BMI} \geqslant 30 \mathrm{~kg} \cdot \mathrm{m}^{-2}$. Level of education was categorised as $\leqslant 12$ years or $>12$ years of formal education. Total numbers are lower than the 806 included participants because some individuals had missing information on covariates. HR: hazard ratio; CMV: cytomegalovirus; $F E V_{1}$ : forced expiratory volume in $1 \mathrm{~s}$. \#: includes death events with COPD listed as either the underlying cause or a coexisting condition on the death certificate. 
pathogens C. pneumoniae and M. pneumoniae, and systemic inflammation (i.e. serum levels of CRP). Similar effects of CMV serology on mortality risk were also confirmed in the group of participants who had already the hallmark of COPD, i.e. airflow limitation, at enrolment.

Growing evidence supports a possible role of chronic viral infections in COPD development [25]. In this context, the association of CMV with COPD mortality may be the consequence of direct effects of the virus in the lung, a common site of viral reactivation [26]. This scenario would be consistent with recent observations that CMV may accelerate lung disease progression in cystic fibrosis [27]. Lung function deficits in COPD may also originate in response to interactions between the virus and environmental triggers of the disease - first and foremost, cigarette smoking - although in our study, we did not observe an increased effect of CMV on COPD mortality among smokers. Alternatively, CMV may influence COPD mortality through its effects on chronic inflammation and predisposition to infections, which are both well-documented in CMV-infected patients [4-7] and recognised as key players in COPD pathogenesis $[21,22]$. Indeed, recently, anti-CMV antibody levels were found to be higher in patients with COPD than in controls, and a positive correlation was observed between CMV serology with soluble tumour necrosis factor receptor-1 levels, a marker of systemic inflammation, and with circulating CD28 ${ }^{\text {null }}$ T-cells, a marker of immune ageing [18]. Although we did find a positive correlation between CMV serology and immune responses to the two lung pathogens C. pneumoniae $[28,29]$ and M. pneumoniae [29], in our study, the effects of CMV on COPD mortality were neither modified nor confounded by the inclusion of C. pneumoniae and M. pneumoniae in Cox models. Indeed, in the final Cox model (table 4), both CMV and C. pneumoniae serology had independent, significant effects on COPD mortality. Similarly, although previous studies reported synergistic effects of CMV and systemic inflammation on all-cause mortality risk $[8,11]$, we did not observe such interaction in our analyses on COPD mortality, at least when systemic inflammation was assessed by CRP levels at a single point in time.

Previous studies have provided variable estimates for the association of CMV serology with all-cause mortality risk [9-11, 13-15]. These discrepancies may be partially explained by differences in the way CMV serology has been analysed. Simply comparing CMV seropositivity to seronegativity may capture only a marginal mortality risk increment [11, 13-15], while analysing CMV serology on a quantitative scale may provide stronger power $[9,10,13,14]$. In addition, the highly significant CMV-by-age interaction in our study indicates stronger effects of CMV on mortality risk in younger than older adults and this can also explain some discrepant results from previous studies that differed substantially in the age distribution of their populations. While the presence of a negative or low CMV serology in older age may characterise individuals with a unique resilience to the virus and strong predisposition to longevity [15], the results of our study suggest that a high CMV serology may be a more specific marker of mortality risk when it is present at younger ages, as shown by the steep slope of the estimated hazard ratios for the association of high CMV serology with all-cause mortality and with COPD mortality in particular (figures 2 and S1). Indeed, the proportion of individuals with high CMV serology was substantially lower among participants aged $<55$ years than among participants $\geqslant 55$ years $(26 \%$ versus $41 \%$, respectively), suggesting that high CMV among young participants may identify a specific group of individuals susceptible to earlier and/or more frequent reactivations of CMV.

A relation between CMV and cardiovascular mortality has been previously sought but not unequivocally demonstrated $[9,11,13]$. The observation that in our study, CMV serology was not associated with cardiovascular mortality risk, in contrast to COPD mortality, suggests that CMV may affect the lung preferentially to other organs. We also found that the effects of high CMV serology on cancer mortality were mainly related to specific malignancies, such as urogenital cancers, possibly related to specific tissue tropism of the virus [30].

This study has certain limitations. We evaluated CMV serology at a single time point and could not directly demonstrate the presence of CMV reactivation. However, observations of high CMV antibody titre in subjects with reactivation [31] suggest that high CMV serology identifies subjects with longer and/or more frequent reactivations. Because of the relatively small sample size of some of the cause-specific mortality analyses, we cannot exclude that some of the negative findings may have resulted from insufficient statistical power. We also could not determine to what extent lung function abnormalities were reversible because, like most other large epidemiological cohorts, TESAOD did not include lung function tests after bronchodilator at enrolment.

Among the strengths of our study are the collection of serological data in an adult cohort, the population-based nature and broad age range of the TESAOD population, and the availability of extensive data on mortality events collected over 45 years of follow-up.

In conclusion, in this study, we report a relation between high CMV serology and the subsequent risk of dying from COPD, thus identifying a novel risk factor for COPD mortality. 
Author contributions: R. Nenna, F.D. Martinez, M. Halonen and S. Guerra contributed to the study concept and design. S. Guerra, F.D. Martinez and M. Halonen obtained funding. A. Spangenberg and M. Halonen performed the assays. R. Nenna, J. Zhai, D.L. Sherrill and S. Guerra performed the statistical analysis. J. Zhai and S.E. Packard aided in collection of and provided access to data for analysis. R. Nenna and S. Guerra wrote the manuscript with input from all authors. R. Nenna, J. Zhai, S.E. Packard, A. Spangenberg, D.L. Sherrill, F.D. Martinez, M. Halonen and S. Guerra reviewed the manuscript.

Support statement: This work was supported by grant awards P50 HL107188 and R01 HL095021 from the National Heart, Lung, and Blood Institute. Funding information for this article has been deposited with the Crossref Funder Registry.

Conflict of interest: R. Nenna has nothing to disclose. J. Zhai has nothing to disclose. S.E. Packard reports grants from the NIH during the conduct of the study. A. Spangenberg has nothing to disclose. D.L. Sherrill has nothing to disclose F.D. Martinez reports grants HL139054, HL132523, HL091889, HL130045, HL098112 and HL056177 from NIH/ NHLBI, grant ES006614 from NIH/NIEHS, grant AI126614 from NIH/NIAID, grant OD023282 from NIH/Office of Director, grant UA009253-0001 from Johnson \& Johnson, and personal fees for consultancy from Copeval and Commense Inc., outside the submitted work. M. Halonen reports grants from the NIH during the conduct of the study. S. Guerra reports grants from the NIH during the conduct of the study.

\section{References}

1 Zuhair M, Smit GSA, Wallis G, et al. Estimation of the worldwide seroprevalence of cytomegalovirus: a systematic review and meta-analysis. Rev Med Virol 2019; 29: e2034.

2 Bate SL, Dollard SC, Cannon MJ. Cytomegalovirus seroprevalence in the United States: the national health and nutrition examination surveys, 1988-2004. Clin Infect Dis 2010; 50: 1439-1447.

3 McVoy MA, Adler SP. Immunologic evidence for frequent age-related cytomegalovirus reactivation in seropositive immunocompetent individuals. J Infect Dis 1989; 160: 1-10.

4 Radunovic M, Tomanovic N, Novakovic I, et al. Cytomegalovirus induces Interleukin-6 mediated inflammatory response in salivary gland cancer. J Buon 2016; 21: 1530-1536.

5 Di Benedetto S, Gaetjen M, Muller L. The modulatory effect of gender and cytomegalovirus-seropositivity on circulating inflammatory factors and cognitive performance in elderly individuals. Int J Mol Sci 2019; 20: E990.

6 Chidrawar S, Khan N, Wei W, et al. Cytomegalovirus-seropositivity has a profound influence on the magnitude of major lymphoid subsets within healthy individuals. Clin Exp Immunol 2009; 155: 423-432.

7 Hadrup SR, Strindhall J, Kollgaard T, et al. Longitudinal studies of clonally expanded CD8 T cells reveal a repertoire shrinkage predicting mortality and an increased number of dysfunctional cytomegalovirus-specific $\mathrm{T}$ cells in the very elderly. J Immunol 2006; 176: 2645-2653.

8 Muhlestein JB, Horne BD, Carlquist JF, et al. Cytomegalovirus seropositivity and C-reactive protein have independent and combined predictive value for mortality in patients with angiographically demonstrated coronary artery disease. Circulation 2000; 102: 1917-1923.

9 Roberts ET, Haan MN, Dowd JB, et al. Cytomegalovirus antibody levels, inflammation, and mortality among elderly Latinos over 9 years of follow-up. Am J Epidemiol 2010; 172: 363-371.

10 Wang GC, Kao WH, Murakami P, et al. Cytomegalovirus infection and the risk of mortality and frailty in older women: a prospective observational cohort study. Am J Epidemiol 2010; 171: 1144-1152.

11 Simanek AM, Dowd JB, Pawelec G, et al. Seropositivity to cytomegalovirus, inflammation, all-cause and cardiovascular disease-related mortality in the United States. PLoS One 2011; 6: e16103.

12 Savva GM, Pachnio A, Kaul B, et al. Cytomegalovirus infection is associated with increased mortality in the older population. Aging Cell 2013; 12: 381-387.

13 Gkrania-Klotsas E, Langenberg C, Sharp SJ, et al. Seropositivity and higher immunoglobulin G antibody levels against cytomegalovirus are associated with mortality in the population-based European Prospective Investigation of Cancer-Norfolk cohort. Clin Infect Dis 2013; 56: 1421-1427.

14 Mathei C, Adriaensen W, Vaes B, et al. No relation between CMV infection and mortality in the oldest old: results from the Belfrail study. Age Ageing 2015; 44: 130-135.

15 Feinstein L, Douglas CE, Stebbins RC, et al. Does cytomegalovirus infection contribute to socioeconomic disparities in all-cause mortality? Mech Ageing Dev 2016; 158: 53-61.

16 Adler SP, Best AM, Marshall B, et al. Infection with cytomegalovirus is not associated with premature mortality. Infect Dis Rep 2011; 3: e17.

17 Haeseker MB, Pijpers E, Dukers-Muijrers NH, et al. Association of cytomegalovirus and other pathogens with frailty and diabetes mellitus, but not with cardiovascular disease and mortality in psycho-geriatric patients; a prospective cohort study. Immun Ageing 2013; 10: 30.

18 Tan DB, Amran FS, Teo TH, et al. Levels of CMV-reactive antibodies correlate with the induction of CD28 ${ }^{\text {null }} \mathrm{T}$ cells and systemic inflammation in chronic obstructive pulmonary disease (COPD). Cell Mol Immunol 2016; 13 551-553.

19 Vogelmeier CF, Criner GJ, Martinez FJ, et al. Global Strategy for the Diagnosis, Management, and Prevention of Chronic Obstructive Lung Disease 2017 Report. GOLD Executive Summary. Am J Respir Crit Care Med 2017; 195: 557-582.

20 Lange P, Celli B, Agusti A, et al. Lung-function trajectories leading to chronic obstructive pulmonary disease. N Engl J Med 2015; 373: 111-122.

21 Sethi S, Mahler DA, Marcus P, et al. Inflammation in COPD: implications for management. Am J Med 2012; 125: $1162-1170$.

22 Matkovic Z, Miravitlles M. Chronic bronchial infection in COPD. Is there an infective phenotype? Respir Med 2013; 107: 10-22.

23 Lebowitz MD, Knudson RJ, Burrows B. Tucson epidemiologic study of obstructive lung diseases. I: Methodology and prevalence of disease. Am J Epidemiol 1975; 102: 137-152. 
24 Knudson RJ, Lebowitz MD, Holberg CJ, et al. Changes in the normal maximal expiratory flow-volume curve with growth and aging. Am Rev Respir Dis 1983; 127: 725-734.

25 Linden D, Guo-Parke H, Coyle PV, et al. Respiratory viral infection: a potential "missing link" in the pathogenesis of COPD. Eur Respir Rev 2019; 28: 180063.

26 Cook $\mathrm{CH}$, Zhang Y, Sedmak DD, et al. Pulmonary cytomegalovirus reactivation causes pathology in immunocompetent mice. Crit Care Med 2006; 34: 842-849.

27 Parkins MD, Ramos KJ, Goss $\mathrm{CH}$, et al. Cytomegalovirus: an unrecognised potential contributor to cystic fibrosis disease progression? Eur Respir J 2019; 53: 1801727.

28 Blasi F, Damato S, Cosentini R, et al. Chlamydia pneumoniae and chronic bronchitis: association with severity and bacterial clearance following treatment. Thorax 2002; 57: 672-676.

29 Muro S, Tabara Y, Matsumoto H, et al. Relationship among Chlamydia and Mycoplasma Pneumoniae seropositivity, IKZF1 genotype and chronic obstructive pulmonary disease in a general Japanese population: the Nagahama Study. Medicine (Baltimore) 2016; 95: e3371.

30 Panagiotakis GI, Papadogianni D, Chatziioannou MN, et al. Association of human herpes, papilloma and polyoma virus families with bladder cancer. Tumour Biol 2013; 34: 71-79.

31 Mehta SK, Stowe RP, Feiveson AH, et al. Reactivation and shedding of cytomegalovirus in astronauts during spaceflight. J Infect Dis 2000; 182: 1761-1764. 